\title{
Correlation analysis of Trial of Org 10172 in acute stroke treatment classification and National Institutes of Health Stroke Scale score in acute cerebral infarction with risk factors
}

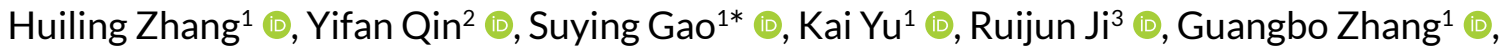 \\ Xifeng Yao ${ }^{1}$ (1) , Jie Bai ${ }^{1}$ (1), Dongliang Liu ${ }^{1}$ ()
}

\begin{abstract}
SUMMARY
OBJECTIVE: The aim of this study was to investigate the correlation between the Trial of Org 10172 in acute stroke treatment classification and the National Institutes of Health Stroke Scale score of acute cerebral infarction as well as acute cerebral infarction's risk factors.

METHODS: The clinical data of 3,996 patients with acute cerebral infarction hospitalized in Hebei Renqiu Kangjixintu Hospital from January 2014 to November 2018 were analyzed retrospectively. According to Trial of Org 10172 in acute stroke treatment, they were divided into five groups: arteriosclerosis, cardio cerebral embolism, arterial occlusion, other causes, and unknown causes. Through questionnaire design, routine physical examination, and physical and chemical analysis of fasting venous blood samples, the risk factors were evaluated, and the correlation between Trial of Org 10172 in acute stroke treatment classification and National Institutes of Health Stroke Scale classification was analyzed using multivariate logistic regression. In addition, the relationship between National Institutes of Health Stroke Scale score and risk factors in different groups was compared, and the correlation between Trial of Org 10172 in acute stroke treatment classification and National Institutes of Health Stroke Scale score was analyzed. RESULTS: Multivariate logistic regression analysis showed that diabetes, atrial fibrillation or stroke history, age, and education level were related to Trial of Org 10172 in acute stroke treatment classification. In the National Institutes of Health Stroke Scale comparison, the scores of the cardio cerebral embolism group were significantly higher than those of the other four groups, and patients with diabetes, atrial fibrillation, or stroke history had a high share, especially atrial fibrillation (33.06\%).

CONCLUSIONS: The nerve function defect is more serious after acute cerebral infarction with cardiogenic cerebral embolism, indicating a poor prognosis. KEYWORDS: Cerebral infarction. Ischemic stroke. Classification. Risk factors. Cerebrovascular.
\end{abstract}

\section{INTRODUCTION}

Acute cerebral infarction (ACI) is a cerebrovascular disease with high morbidity, disability, and mortality ${ }^{1}$ that is affected by various factors. The pathological basis of ACI is the disruption of blood supply in the brain, with ischemia and hypoxia causing ischemic necrosis and cerebral malacia in focal brain tissue ${ }^{2}$. The trial of Org 10172 in acute stroke treatment (TOAST) and the National Institutes of Health Stroke Scale (NIHSS) are currently the most commonly used ACI typing and scoring methods $^{3}$. The TOAST classification denotes five subtypes (LAA, CE, $\mathrm{SAO}, \mathrm{OE}, \mathrm{UE}$ ) of ischemic stroke. LAA patients have large-artery atherosclerosis (embolus/thrombosis), CE is the cardioembolism, SAO patients were defined as having small-vessel occlusion, and $\mathrm{OE}$ and UE mean a stroke of other determined etiology and a stroke of undetermined etiology respectively, and the specific standard refers to the classification method of You Wenxia et al. ${ }^{4}$

NIHSS can comprehensively evaluate the dysfunction after stroke, with objective evaluation criteria and strong operability.
Some scholars ${ }^{5}$ found that the predictive ability of NIHSS changes in the prognostic outcome of ACI patients is excellent. The lower the NIHSS score, the better the prognosis of ACI patients. Furthermore, in several studies, such as those conducted by $\operatorname{Tan}^{6}$ and Wang ${ }^{7}$, by dynamically observing NIHSS changes during the hospitalization of patients of each subtype, it was found that patients with $\mathrm{CE}$ had the most severe neurological deficits and the worst prognosis; conversely, patients with SAO had the least severe neurological deficits and the best prognosis. In the study conducted by Wang ${ }^{8}$, it was also shown that patients with CE had a significantly higher neurological deficit score on admission than other subtypes, a greater proportion of severe cases, a significantly lower rate of neurological improvement at discharge than other subtypes, and the lowest clinical outcome. Moreover, SAO had the lowest neurological deficit score on admission, the mildest disease, the highest rate of neurological improvement at discharge, and the best outcome. In many previous studies, it was suggested that the CE type of cerebral

\footnotetext{
${ }^{1}$ Renqiu Kangjixintu Hospital, Department of neurology - Renqiu, China. ${ }^{2}$ Hebei Medical University, School of Basic Medicine - Shijiazhuang, China. ${ }^{3}$ Capital Medical University, Beijing Tiantan Hospital - Beijing, China.

*Corresponding author: gaosy202010@126.com

Conflicts of interest: the authors declare there is no conflicts of interest. Funding: 2021 Hebei Province Medical Science Research Project Plan. Project No.: 20210705, Subject: Predictive value of pressure ulcer score and fall score on discharge outcome of acute ischemic stroke.

Received on October 09, 2021. Accepted on October 18, 2021.
} 
infarction has a large lesion area, the lesion affects a wide range of patients, the embolism in the brain is mainly caused by the blockage of large arteries by emboli, and the neuroendocrine changes in the acute phase increase the ventricular load, which makes the construction of collateral circulation more difficult. Therefore, the incidence of poor prognosis is high ${ }^{9}$, which has also been confirmed by the autopsy results of patients with ischemic stroke. The SAO type of cerebral infarction is mainly due to luminal changes caused by lipid hyaline degeneration and lumen occlusion in the walls of small arteries and micro-arteries due to long-term hypertension; however, the lesion involvement is small, and the damage is mild ${ }^{10}$. Yuan et al. ${ }^{11}$ compared the results of TOAST and NIHSS with the changes in actual clinical symptoms of the patients, and the analysis showed that NIHSS was a good indicator of the prognosis of the patients.

However, the correlation between ACI TOAST and NIHSS score remains unknown recently. Therefore, we conducted this study to investigate the correlation between ACI TOAST and NIHSS, as well as ACI's risk factors.

\section{METHODS}

\section{Study subjects}

This was a retrospective study. From January 2014 to November 2018, 3,996 patients with ACI TOAST classification who were hospitalized were recruited and divided into five groups based on artery atherosclerosis, cardiac cerebral embolism, SAO, for other reasons, and unknown reasons. This study was conducted in accordance with the Declaration of Helsinki and approved by the ethics committee of our hospital. All participants had signed the informed consent.

\section{Inclusion and exclusion criteria}

Inclusion criteria were

1. patients who had been diagnosed with ACI according to the revised diagnostic criteria of the Chinese Guidelines for the Diagnosis and Treatment of Acute Ischemic Cerebral Stroke $2014^{12}$ and confirmed by cranial CT or MRI;

2. age was more than 18 years;

3. within seven days of stroke onset; and

4. patients who have signed informed consent.

Exclusion criteria were

1. patients with severe consciousness disorders, psychiatric symptoms, and aphasia;

2. patients who had a hearing impairment and could not cooperate with the examination;
3. patients with hemorrhagic cerebrovascular disease;

4. patients who had the previous history of psychiatric and psychological diseases;

5. patients with combined chronic wasting diseases, malignant tumors, hyperthyroidism, or hematologic diseases; and

6. patients with autoimmune system diseases.

\section{Methods}

All the data were investigated by uniformly trained medical personnel in accordance with a uniformly designed questionnaire for eligible patients. The study included a questionnaire, physical examination, laboratory examination, and discharge-related data collection and entry. This study was conducted in accordance with the Declaration of Helsinki and approved by the ethics committee of Renqiu Kangjixintu Hospital.

\section{Questionnaire}

This questionnaire included age, gender, and risk factors for vascular diseases, such as the history of hypertension, diabetes, hyperlipidemia, stroke, coronary artery disease, atrial fibrillation, and the use of antiplatelet drugs.

\section{Physical examination}

The physical examination included blood pressure, heart rate, height, weight, and waist circumference. For blood pressure and heart rate measurements, all respondents were asked to sit still for 5-10 min before the examination, and then an Omron electronic blood pressure monitor was used to measure the systolic and diastolic blood pressure of the upper extremities of the selected subjects bilaterally. The highest side systolic and diastolic blood pressure was taken from twice examinations for measurement as the final result record.

\section{Laboratory examination}

After fasting for $12 \mathrm{~h}, 6 \mathrm{~mL}$ of fasting venous blood specimens were routinely collected from all the participants, and the fibrinogen (FIB) was measured using a Succeeder SF-8000. The fasting plasma glucose (FPG), total cholesterol (TC), triglyceride (TG), low-density lipoprotein cholesterol (LDL-C), high-density lipoprotein cholesterol (HDL-C), lipoprotein a, homocysteine ( $\mathrm{HCY}$ ), uric acid (UA), cystatin C, urea nitrogen, and creatinine were measured using an Olympus 400 fully automated biochemical detector.

\section{TOAST etiological typing}

Based on TOAST, ischemic stroke was classified into five types: large artery atherosclerosis (LAA), cardioembolism (CE), small 
artery occlusion (SAO), other determined etiologies (OE), and unexplained etiology (UE).

\section{Statistical Methods}

All statistical analyses were performed using SPSS version 21.0 (IBM, Chicago, USA). The continuous variables of normal distribution were expressed as mean \pm standard deviation, the continuous variables of non-normal distribution were expressed as median (interquartile range [IQR]), and the categorical variables were expressed as frequency (percentage [\%]). For multiple comparisons, each value was compared using oneway ANOVA following Dunnett's test when each datum conformed to the normal distribution, while the non-normally distributed continuous data were compared using non-parametric tests. The counting data were tested by chi-square test. Multivariate logistic regression was used to determine the independent variable in this study. A value of $\mathrm{p}<0.05$ was considered statistically significant.

\section{RESULTS}

\section{Baseline characteristics}

These participants had been divided into five groups, such as LAA group (3,338 cases), CE group (124 cases), SAO group (489 cases), OE group (24 cases), and UE group (21 cases).

\section{Comparison outcomes among the five groups}

The results showed that there were statistically significant differences among the five groups in terms of education, age, history of hypertension, diabetes, hyperlipidemia, history of stroke, atrial fibrillation, coronary artery disease, whether antiplatelet agents were used before the disease, systolic blood pressure, diastolic blood pressure, FPG, UA, and urea nitrogen levels on admission $(\mathrm{p}<0.05)$. However, there were no statistically significant differences among the five groups in terms of gender, body mass index, smoking, excessive alcohol consumption, whether the peripheral vascular disease was combined, FIB, TG, TC, LDL-C, HDL-C, lipoprotein a, HCY, creatinine, and cystatin C levels on admission ( $p>0.05$ ) (Table 1$)$.

\section{National Institutes of Health Stroke Scale in patients with acute cerebral infarction Trial of Org 10172 in acute stroke treatment on admission}

The results showed that the NIHSS on admission were $3.61 \pm 3.56$ in the LAA group, 5.69 \pm 6.14 in the CE group, $2.55 \pm 2.06$ in the SAO group, 3.54 \pm 3.91 in the OE group, and $3.10 \pm 3.33$ in the UE group. In pairwise comparisons, the difference between the LAA and CE, LAA and SAO, $\mathrm{CE}$ and $\mathrm{SAO}$, and SAO and OE was statistically significant $(\mathrm{p}<0.05)$. However, no statistically significant differences were observed between/among other comparison groups (Table 2).

\section{National Institutes of Health Stroke Scale in patients with acute cerebral infarction Trial of Org 10172 in acute stroke treatment at discharge}

The results showed that the NIHSS at discharge were 3.13 \pm 3.69 in the LAA group, 4.39 \pm 5.82 in the CE group, $2.00 \pm 2.03$ in the SAO group, 3.21 \pm 4.28 in the $\mathrm{OE}$ group, and $2.43,3.21 \pm 4$ in the UE group. In pairwise comparisons, the difference between the LAA and CE, LAA and SAO, CE and SAO, and SAO and OE was statistically significant $(\mathrm{p}<0.05)$. However, no statistically significant differences were observed between/among other comparison groups (Table 2).

\section{Analysis of Trial of Org 10172 in acute stroke treatment classification}

The results of multivariate logistic regression analysis of TOAST classification are given in Table 3. In multivariate logistic regression analysis, patients with diabetes, atrial fibrillation, or a history of stroke were more likely and had the risk of ACI, besides these factors played a major effect on the TOAST classification (Table 3). There were no episodes of TOAST classification associated with gender. Age and culture degree had a relationship with TOAST classification by the multivariate logistic regression analysis.

\section{DISCUSSION}

In this study, according to the TOAST of ACI, LAA cerebral infarction was the most frequent, followed by $\mathrm{SAO}$ and $\mathrm{CE}$, while $\mathrm{OE}$ and $\mathrm{CE}$ caused the least $\mathrm{ACI}$, which is basically consistent with the findings of $\mathrm{Han}^{13}$ and Jiang ${ }^{14}$. Lei et al. ${ }^{15}$ found that the top three were $\mathrm{UE}, \mathrm{SAO}$, and $\mathrm{CE}$, while $\mathrm{Li}$ et al. ${ }^{16}$ found that the top three were LAA, SAO, and CE. The proportion of various subtypes varied in different studies, and the reasons for this may be related to the differences in sample size, geography, examination methods, and many other factors.

In this study, it was found that the patient's education, age, whether combined with hypertension, diabetes mellitus, hyperlipidemia, history of stroke, atrial fibrillation, coronary artery disease, whether antiplatelet drugs were used before the disease, 
Table 1. Baseline information of TOAST etiological subgroups of $\mathrm{ACl}$.

\begin{tabular}{|c|c|c|c|c|c|c|c|}
\hline Items & $\begin{array}{c}\text { LAA } \\
(3,338 \text { cases })\end{array}$ & $\begin{array}{c}\text { CE } \\
\text { (124 cases) }\end{array}$ & $\begin{array}{c}\text { SAO } \\
\text { (489 cases) }\end{array}$ & $\begin{array}{c}\text { OE } \\
\text { (24 cases) }\end{array}$ & $\begin{array}{c}\text { UE } \\
\text { (21 cases) }\end{array}$ & $\begin{array}{l}\text { Test statistic } \\
\text { value }\end{array}$ & p-value \\
\hline \multicolumn{8}{|l|}{ Demographic characteristics } \\
\hline Male [cases (\%)] & $2,053(61.50)$ & $82(6.13)$ & $318(65.03)$ & $13(54.17)$ & 15 (71.43) & 4.561 & 0.335 \\
\hline $\begin{array}{l}\text { Junior high school and } \\
\text { higher education }\end{array}$ & $889(26.63)$ & $33(26.61)$ & $195(39.880$ & $10(41.67)$ & $14(66.67)$ & 53.978 & 0.000 \\
\hline Age (years) & $65.62 \pm 10.88$ & $68.10 \pm 12.74$ & $59.84 \pm 11.07$ & $52.33 \pm 13.38$ & $50.57 \pm 16.28$ & 48.658 & 0.000 \\
\hline Body mass index & $24.92 \pm 3.63$ & $24.94 \pm 3.40$ & $24.92 \pm 3.47$ & $25.06 \pm 4.41$ & $24.06 \pm 3.39$ & 0.310 & 0.871 \\
\hline \multicolumn{8}{|l|}{ Risk factors n (\%) } \\
\hline Hypertension & 2,372 (71.06) & $73(58.87)$ & $321(65.64)$ & $12(50.00)$ & $13(61.90)$ & 18.645 & 0.001 \\
\hline Diabetes & $684(20.49)$ & $15(12.10)$ & $68(13.91)$ & $3(12.50)$ & $2(9.52)$ & 18.294 & 0.001 \\
\hline Hyperlipidemia & 465 (13.93) & $20(16.13)$ & $40(8.18)$ & $2(8.33)$ & $2(9.52)$ & 13.943 & 0.007 \\
\hline History of stroke & 1,201 (35.98) & $44(35.48)$ & $94(19.22)$ & $7(29.17)$ & $3(14.29)$ & 57.518 & 0.000 \\
\hline Atrial fibrillation & $45(1.35)$ & $41(33.06)$ & $4(0.82)$ & $0(0.00)$ & $0(0.00)$ & 552.744 & 0.000 \\
\hline Coronary heart disease & 455 (13.63) & $45(36.29)$ & $38(7.77)$ & $2(8.33)$ & $4(19.05)$ & 69.512 & 0.000 \\
\hline Smoking & $1,608(48.17)$ & $47(37.90)$ & $222(45.40)$ & $13(54.17)$ & $7(33.33)$ & 8.168 & 0.086 \\
\hline $\begin{array}{l}\text { Excessive alcohol } \\
\text { consumption }\end{array}$ & $146(4.37)$ & $3(2.42)$ & $28(5.73)$ & $\mathrm{O}(0.00)$ & $1(4.76)$ & 4.239 & 0.375 \\
\hline Antiplatelet drug use & 547 (16.39) & $31(25.00)$ & $48(9.82)$ & $1(4.17)$ & $2(9.53)$ & 25.045 & 0.000 \\
\hline $\begin{array}{l}\text { Peripheral } \\
\text { vascular disease }\end{array}$ & $12(0.36)$ & $0(0.00)$ & $2(0.41)$ & $0(0.00)$ & $0(0.00)$ & 0.650 & 0.957 \\
\hline $\begin{array}{l}\text { Systolic blood pressure } \\
(\mathrm{mmHg})\end{array}$ & $164.15 \pm 24.18$ & $158.17 \pm 25.58$ & $161.92 \pm 24.02$ & $151.38 \pm 29.23$ & $154.62 \pm 26.75$ & 4.808 & 0.001 \\
\hline $\begin{array}{l}\text { Diastolic blood } \\
\text { pressure }(\mathrm{mmHg})\end{array}$ & $87.59 \pm 13.31$ & $92.84 \pm 16.85$ & $90.75 \pm 13.39$ & $85.79 \pm 15.06$ & $90.14 \pm 11.52$ & 10.071 & 0.000 \\
\hline $\mathrm{FIB}(\mathrm{g} / \mathrm{L})$ & $3,11 \pm 1.59$ & $3.07 \pm 1.18$ & $2.93 \pm 1.21$ & $3.12 \pm 0.73$ & $2.82 \pm 0.79$ & 1.743 & 0.138 \\
\hline $\mathrm{FPG}(\mathrm{mmol} / \mathrm{L})$ & $6.22 \pm 2.37$ & $5.98 \pm 2.02$ & $5.79 \pm 1.89$ & $5.21 \pm 3.39$ & $5.62 \pm 1.85$ & 4.171 & 0.002 \\
\hline $\mathrm{TG}(\mathrm{mmol} / \mathrm{L})$ & $1.54 \pm 1.42$ & $1.35 \pm 1.08$ & $1.57 \pm 1.29$ & $1.40 \pm 0.69$ & $1.37 \pm 0.65$ & 0.811 & 0.518 \\
\hline $\mathrm{TC}(\mathrm{mmol} / \mathrm{L})$ & $4.81 \pm 1.15$ & $4.76 \pm 1.21$ & $4.71 \pm 1.07$ & $4.89 \pm 1.04$ & $4.45 \pm 1.08$ & 1.495 & 0.201 \\
\hline LDL-C (mmol/L) & $2.72 \pm 7.14$ & $2.35 \pm 0.80$ & $2.51 \pm 5.26$ & $2.42 \pm 0.69$ & $2.32 \pm 0.67$ & 0.208 & 0.934 \\
\hline HDL-C (umol/L) & $1.18 \pm 2.17$ & $1,12 \pm 0.30$ & $1.16 \pm 0.33$ & $1.03 \pm 0.38$ & $1.10 \pm 0.26$ & 0.080 & 0.988 \\
\hline Lipoprotein a (mg/L) & $272.38 \pm 264.69$ & $272.93 \pm 239.08$ & $248.76 \pm 366.96$ & $282.20 \pm 185.51$ & $357.98 \pm 420.77$ & 1.306 & 0.265 \\
\hline $\mathrm{HCY}(\mathrm{mmol} / / \mathrm{L})$ & $20.19 \pm 15.27$ & $21.29 \pm 15.62$ & $19.58 \pm 17.39$ & $17.75 \pm 12.99$ & $21.99 \pm 15.96$ & 0.552 & 0.697 \\
\hline UA (umol/L) & $334.20 \pm 102.12$ & $361.42 \pm 112.52$ & $331.65 \pm 96.99$ & $316.02 \pm 131.41$ & $338.16 \pm 104.23$ & 2.462 & 0.043 \\
\hline $\begin{array}{l}\text { Urea nitrogen } \\
(\mathrm{mmol} / / \mathrm{L})\end{array}$ & $5.71 \pm 2.07$ & $6.68 \pm 7.36$ & $5.44 \pm 1.67$ & $5.45 \pm 2.09$ & $5.34 \pm 1.27$ & 6.948 & 0.000 \\
\hline Creatinine (umol/L) & $71.56 \pm 33.49$ & $76.00 \pm 23.99$ & $68.72 \pm 33.44$ & $67.90 \pm 21.99$ & $63.68 \pm 15.47$ & 1.761 & 0.134 \\
\hline Cystatin C (mg/L) & $1.13 \pm 6.55$ & $1.06 \pm 0.59$ & $0.87 \pm 0.43$ & $0.83 \pm 0.44$ & $0.84 \pm 0.33$ & 0.221 & 0.927 \\
\hline
\end{tabular}

systolic blood pressure, diastolic blood pressure, FPG, UA, and urea nitrogen levels on admission were factors that were associated with ACI. In addition, the NIHSS scores on admission and at discharge were compared among the five groups after grouping ACI patients according to TOAST. The data showed that the NIHSS scores were the highest in the CE group and the lowest in the SAO group, suggesting that the CE group had more severe neurological deficits and the worst prognosis in this study, which could be explained that the neuroendocrine changes in the brain embolism increase the ventricular 
Table 2. Comparison of NIHSS in patients with ACI TOAST.

\begin{tabular}{|c|c|c|c|c|c|c|}
\hline Groups & LAA & CE & SAO & OE & \multicolumn{2}{|c|}{ UE } \\
\hline NIHSS at admission & $3.61 \mathrm{~S}$ at & $5.69 \mathrm{~S}$ at & $2.55 \mathrm{~S}$ at & $3.54 \mathrm{~S}$ at & \multicolumn{2}{|c|}{$3.10 \mathrm{~S}$ at } \\
\hline \multirow[t]{3}{*}{ NIHSS at discharge } & $3.13 \mathrm{~S}$ at & $4.39 S$ at & $2.00 S$ at & $3.21 \mathrm{~S}$ at & & \\
\hline & \multirow{2}{*}{ Comparisons } & \multirow{2}{*}{$\begin{array}{c}\text { Mean } \\
\text { differences } \\
(I-J)\end{array}$} & \multirow{2}{*}{ Standard error } & \multirow{2}{*}{ Sig. } & \multicolumn{2}{|c|}{ 95\% Confidence interval } \\
\hline & & & & & Lower bound & Upper bound \\
\hline \multirow{5}{*}{ NIHSS at admission } & LAA V.S. CE & -2.07941 & 0.32203 & 0.000 & -2.9582 & -1.2006 \\
\hline & LAA V.S. SAO & 1.06813 & 0.17050 & 0.000 & 0.6028 & 1.5334 \\
\hline & CE V.S.SAO & 3.14754 & 0.35404 & 0.000 & 2.1813 & 4.1137 \\
\hline & CE V.S. OE & 2.15188 & 0.78525 & 0.048 & 0.0089 & 4.2948 \\
\hline & CE V.S. UE & 2.59831 & 0.83091 & 0.015 & 0.3307 & 4.8659 \\
\hline \multirow{3}{*}{ NIHSS at discharge } & LAA V.S. CE & -1.25602 & 0.33092 & 0.001 & -2.1591 & -0.3529 \\
\hline & LAA V.S. SAO & 1.13312 & 0.17522 & 0.000 & 0.6549 & 1.6113 \\
\hline & CE V.S.SAO & 2.38914 & 0.36381 & 0.000 & 1.3963 & 3.3820 \\
\hline
\end{tabular}

Table 3. Multivariate logistic regression analysis of TOAST classification.

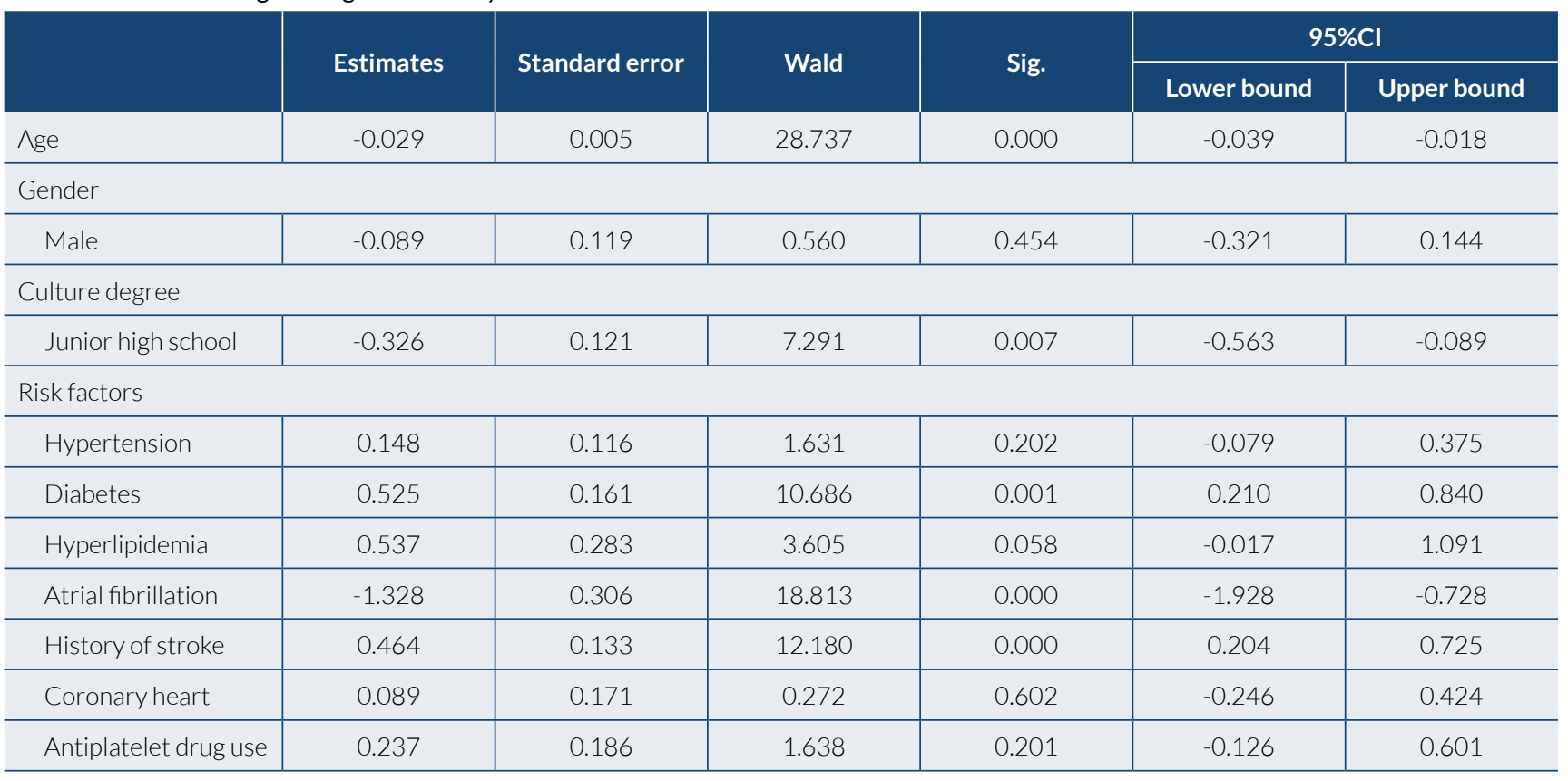

load and make the construction of collateral circulation more difficult ${ }^{9}$. However, there was no significant difference in the NIHSS scores between the other three groups. In the baseline information study of different groups, the first three factors related to TOAST classification, atrial fibrillation, stroke history, and diabetes accounted for a very high proportion in group CE, especially atrial fibrillation, accounting for 33.06\%, much higher than that in other groups, which was consistent with the results of previous studies, indicating the reliability of the research data. In clinical work, we routinely perform the TOAST of ACI patients to a certain extent to predict their prognosis $^{17-19}$.

There were several limitations in this study. Firstly, this trial was not a randomized controlled trial. Secondly, only the NIHSS scores of patients with ACI on admission and at discharge were compared after grouping in this study, and long-term follow-up 
was not performed, which may leave a gap in the judgment of long-term prognosis.

\section{CONCLUSIONS}

The TOAST classification is effective and reliable, and the related factors include diabetes, atrial fibrillation or stroke history, age, and education level. NIHSS score revealed that neurological impairment after ACI combined with cardiogenic cerebral embolism is more serious, suggesting a poor prognosis. This is consistent with the results of TOAST classification results, and the two have a certain correlation, which is worth further study.

\section{REFERENCES}

1. QiaoZhen X, AiGuo M, Tong W, Jing Jing L, HaiYing L. Correlation between of small dense low-density lipoprotein cholesterol with acute cerebral infarction and carotid atherosclerotic plaque stability. J Clin Lab Anal. 2019;33(6):e22891. https://doi.org/10.1002/ jcla.22891

2. Ahlhelm FJ. Intubation und Sedierung bei der endovaskulären Therapie des akuten Hirninfarkts [Intubation and sedation in the endovascular treatment of acute cerebral infarction]. Radiologe. 2016;56(1):42-6. https://doi.org/10.1007/s00117-015-0056-8

3. Chang HJ, Quan YP, Chen SZ. Relationship between TOAST classification and neurological function and prognosis in acute cerebral infarction. Chinese Journal of modern neurological diseases. 2009;9:456-9. https://doi.org/10.3969/j.issn.16726731.2009.05.012

4. You WX, Zhong JP, Ouyang JP, Li HZ, Yang SM, Hu QG. Analysis of the results of SSS-TOAST classification and intracranial infarction in 516 patients with ischemic minor stroke. Shandong Medical Journal. 2017;57(27):9-13. https://doi.org/10.3969/j.issn.1002266X.2017.27.003

5. Qian JT, Jiang JB, Kong ZB. The effect of interventional thrombectomy with solitaire stent in the treatment of acute cerebral infarction and its influence on postoperative complications. Journal of vascular and endovascular surgery. 2018;6:499-503. https://doi. org/CNKI:SUN:XGQW.0.2018-06-006

6. Tan MX, Chen SL, Zhou J. Clinical efficacy analysis of acute ischemic stroke based on toast classification. Chinese and Western Medicine Journal of cerebrovascular disease. 2011;9(08):938-9. https://doi. org/10.3969/j.issn.1672-1349.2011.08.024

7. Wang BX, Xu P. Correlation between TOAST classification and plasma D-dimer in patients with acute ischemic stroke. Journal of stroke and neurological disease. 2010;17(06):354-6. https:// doi.org/10.3969/j.issn.1007-0478.2010.06.011

8. Wang SH, Ren D, Wang QR, Meng B, Zhou XM, Lu JZ. Correlation between serum uric acid and toast classification and early prognosis of acute ischemic stroke. Thrombosis and hemostasis. 2014:4:156158. https://doi.org/10.3969/j.issn.1009-6213.2014.04.002

9. Wu S, Wu B, Liu M, Chen Z, Wang W, Anderson CS, et al. Stroke in China: advances and challenges in epidemiology, prevention, and management. Lancet Neurol. 2019;18(4):394-405. https:// doi.org/10.1016/S1474-4422(18)30500-3

\section{Ethical statement}

This study was conducted in accordance with the Declaration of Helsinki and approved by the ethics committee of our hospital. All participants had signed the informed consent.

\section{AUTHORS' CONTRIBUTIONS}

HZ: Conceptualization. YQ: Conceptualization. SG: Data curation. KY: Data curation. RJ: Data curation. GZ: Formal Analysis. XY: Formal Analysis, Writing - original draft. JB: Formal Analysis, Writing - original draft. DL: Formal Analysis, Writing - original draft.

10. WuY,Hu JF. Correlationanalysis of ischemic stroke and homocysteine, serum uric acid, hs CRP levels. Shandong medicine. 2013;53:80-1. https://doi.org/10.3969/j.issn.1002-266X.2013.29.033

11. Yuan W, Fu NN, Shi ZH. Analysis of risk factors and shortterm prognosis of toast in ischemic stroke. Journal of stroke and neurological disease. 2014;01:53-56. https://doi.org/ CNKI:SUN:ZFSJ.0.2014-01-015

12. Chen XL, Luo DY, LiuW, Huang Y, Liu H. RevisedTOAST classification of ischemic stroke in Korea. Journal of North Sichuan Medical College. 2019; 34(05):504-7. https://doi.org/10.3969/j.issn.10053697.2019.05.04

13. Han LZ, Wang WJ, Wu XY. A comparative study on the clinical characteristics of ischemic stroke in young and elderly. Journal of North Sichuan Medical College. 2019;34(06):779-82. https:// doi.org/10.3969/j.issn.1005-3697.2019.06.32

14. Jiang J, Jiang Y, Feng S, Sun D, Zhuang A, Zeng Q, Zhang Y, Huang H $\mathrm{Nie} \mathrm{H}$, Zhou F. Microembolic signal monitoring of TOAST-classified cerebral infarction patients. Mol Med Rep. 2013;8(4):1135-42. https://doi.org/10.3892/mmr.2013.1609

15. Lei JJ, Wang JFG, Zhang L, Feng K. Study on the relationship between TOAST classification, homocysteine and NIHSS score in patients with acute cerebral infarction. Chinese Journal of practical neurology. 2017;23:9-13. https://doi.org/10.3969/j. issn.1673-5110.2017.23.003

16. Li H, Cai Y, Huang JB, Li Y, Zhi Y. Study on apolipoprotein and plasma lipoprotein-related phospholipase A2 and TOAST etiology classification in patients with ischemic stroke. Clin Focus. 2018;33:298-301.

17. Bao L, Zhang S, Gong X, Cui G. Trousseau Syndrome Related Cerebral Infarction: Clinical Manifestations, Laboratory Findings and Radiological Features. J Stroke Cerebrovasc Dis. 2020;29(9):104891. https://doi.org/10.1016/j.jstrokecerebrovasdis.2020.104891.

18. Terasawa Y, Sakai K, Komatsu T, Sakuta K, Omoto S, Mitsumura H, et al. Microbleeds of lacunar infarction and middle cerebral artery flow velocity of branch atheromatous disease are essential factors of stroke etiology. Eur Neurol. 2019;81(1-2):19-23. https://doi. org/10.1159/000494672

19. Zha M, Li S, Cai HD, Zhao M, Yuan K, Liu R, et al. Therapeutic effect of thrombectomy for acute middle cerebral artery M2 segment occlusion: A preliminary analysis. Chinese Journal of Cerebrovascular Diseases. 2019;16(04):192-6. https://doi. org/10.3969/j.issn.1672-5921.2019.04.005 